\title{
The Incidence of Contrast-Induced Nephropathy in Saudi Population after Cardiac Catheterization
}

\begin{abstract}
Background: Contrast-induced nephropathy CIN is an acute deterioration of renal function defined as the increase in serum creatinine by $25 \%$ above the baseline value within $48 \mathrm{~h}$ after administration of contrast agent, several risk factors may aggravate CIN and DM is a strong predisposing factor.
\end{abstract}

Objectives: To study the effect of contrast administration on renal function, and the relation of diabetes mellitus and other risk factors which can predispose patients to CIN in our local hospital.

Methods: Open-label, single center study. A total of 1117 patients underwent cardiac catheterization between January and December 2009, all patients with acute coronary syndrome, and stable CAD were included.

Results: Among 1117 patients, the incidence of CIN was $(4.9 \%)$ and this represents 55patients. Out of those 55 patients, 35 (64\%) patients were diabetic, $39(71 \%)$ patients were hypertensive, $38(56 \%)$ patients under went diagnostic cardiac catheterization, 17 (34\%) had PCI, 23 (41\%) patients had body weight $<70 \mathrm{~kg}$, and $44(80 \%)$ patients received omnipauqe contrast media.

Conclusion: The incidence of CIN was $4.9 \%$, and this concludes that hypertension and DM are the major risk factors in Saudi patients, diabetic patients in particular should be closely monitored for CIN and preventive measures should be strictly enforced.

Research Article
Volume 10 Issue 1 - 2017
Mohammed Balghith*
King Saud Bin Abdulaziz University for Health Sciences, Saudi
Arabia
*Corresponding author: Mohammed Balghith, King Saud
Bin Abdulaziz University for Health Sciences, Riyadh, P O
Box 24490, Saudi Arabia, Tel: 00966504147204; Email:
mbalghith@hotmail.com
Received: October 27, 2017 | Published: November 07,
2017

\section{Introduction}

Contrast-induced nephropathy (CIN) is the sudden deterioration of kidney function resulting from intravenous (IV) or intra-arterial (IA) administration of contrast media (CM) [1]. Bartels and colleagues first described CIN in 1954 [2]. Since that report, there has been an increased incidence of CIN from the mid-1970s, corresponding with an increase in procedures utilizing contrast administration [1]. Development of contrastinduced nephropathy (CIN), ie, a rise in serum creatinine by either $\geq 0.5 \mathrm{mg} / \mathrm{dL}$ or by $\geq 25 \%$ from baseline within the first $2-3$ days after contrast administration, is strongly associated with both increased in-hospital and late morbidity and mortality after invasive cardiac procedures $[3,4]$.

The prevention of CIN is critical if long-term outcomes are to be optimized after diagnostic or percutaneous coronary intervention (PCI). The prevalence of CIN in patients receiving contrast varies markedly (from $<1 \%$ to $50 \%$ ), depending on the presence of well-known risk factors, the most important of which are baseline chronic renal impairment and diabetes mellitus. Other risk factors include old age, anemia, heart failure, low volume status, dehydration, hypertension, renal transplant, low serum albumin, concomitant use of nephrotoxins, and the volume of contrast agent. Our objective is to study the effect of contrast media administration on kidney function, and the relation of risk factors including diabetes which can predispose patients to CIN.

\section{Methods}

Open-label, single-center study, we include all adult patient aged above 18 years, who came to our cardiac catheterization laboratory (Cath Lab) and underwent either diagnostic or interventional coronary angiography. A total of 1117 patients were included in this study between January and December 2009 , all patients in that period had different indications for their procedure, the majority was admitted with the diagnosis of an Acute coronary syndrome through emergency department.

As a routine practice in our unit, all elective and stable CAD patients will be on IV fluids at least for 24 hours before cardiac catheterization, to have a good hydration prior to contrast media exposure in Cath Lab. The patients with ACS might not have enough time for IV fluids especially Acute STEMI patients who undergo primary PCI. For patients with prior renal impairment and high serum creatinine, they had IV fluids hydration for 2-3 days before their cardiac catheterization.

Measurements of creatinine were done in every patient 1-3 days prior to the procedure, Post-cath creatinine values were taken on the first-day post-procedure, second day and third day. The data was taken according to the patient's medical record number from the hospital computer database and analyzed systematically. We excluded patients with any of the following: (1) Any patients age $<18 \mathrm{yr}$; (2) Patients with ESRD on hemodialysis or peritoneal dialysis; (3) kidney transplant before or planned at 
the time of enrollment. Two contrast media was used, Omnipaque and Visipaque Iohexol (Omnipaque) is a non-ionic, monomer, 350 $\mathrm{mgl} / \mathrm{ml}$ with low osmolality of 884 . The other contrast media was Iodixanol (Visipaque) which is a non-ionic,dimer, $320 \mathrm{mgl} / \mathrm{ml}$ with low osmolality of 290.

\section{Definition of CIN}

Contrast-induced nephropathy was defined as a $25 \%$ increase in the creatinine value from the baseline reading in the $2^{\text {nd }}$ to $3^{\text {rd }}$ day post-cath.

\section{Statistical analysis}

Data were entered in Excel and analyzed using SPSS version 19.0 for Windows (SPSS, Chicago, IL, USA). Demographic characteristics are presented for continuous variables as means and standard deviations and categorical variables as frequencies and percentages. CIN and non-CIN patients were compared using the Chi-square test for categorical variables and Student's t-test for continuous variables. Significance was set at the 0.05 level.

\section{Results}

A total of 1117 patients underwent coronary angiography. The mean age of the study cohort was $55.7( \pm 11.6)$, and $81.4 \%$ were males. The incidence of CIN was (4.9\%) and this represents 55patients (Figure 1). The 1117 patients, underwent two types of procedures, 675 patients underwent diagnostic cath and 442 had PCI (Figure 2). Furthermore, out of the 55 patients who developed CIN a $38(56 \%)$ patient underwent diagnostic cardiac cath, 17 $(34 \%)$ had PCI, and $23(41 \%)$ patients had body weight $<70 \mathrm{~kg}$ In this study two types of contrast media were used, the number of patients who received Omipaquie contrast was 1012, and only 105 received visipuqe. Among the 55 patients who developed contrast nephropathy, the vast majority 44 (80\%) patients, received omnipauqe (Figure 3).

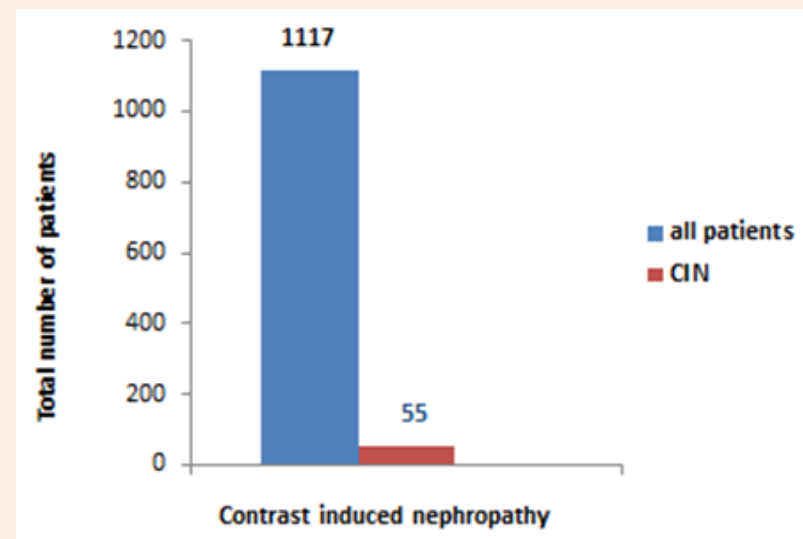

Figure 1: This figure showed that 1117 patients underwent coronary angiography, 55 (4.9\%) of them developed CIN

In those 55 patients, 35 (64\%) patients were diabetic and there was no significant difference in the development of CIN in Diabetic and non-diabetic post-cardiac catheterization, there were 39 (71\%) patients hypertensive, and the difference between those hypertensive and non- hypertensive was statistically significant (Figure 4).

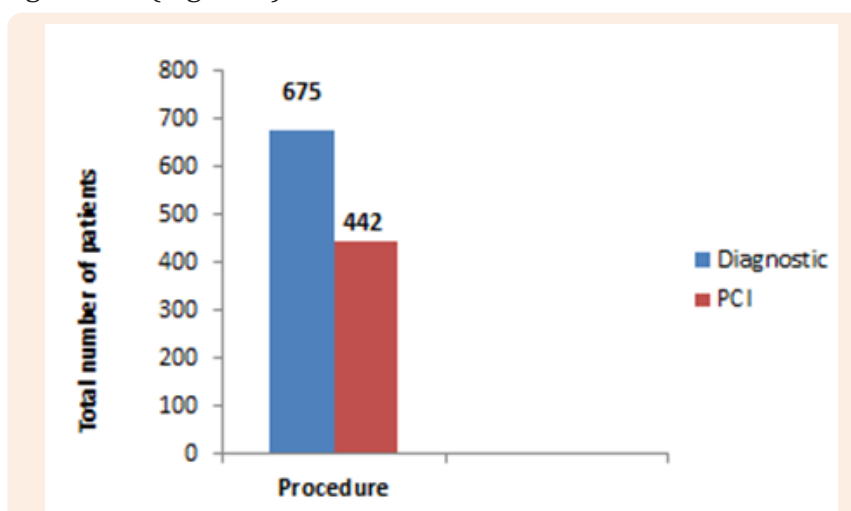

Figure 2: This figure showed the number and types of procedures done to the patients in the study, 675 patients underwent diagnostic cath and 442 had PCI.

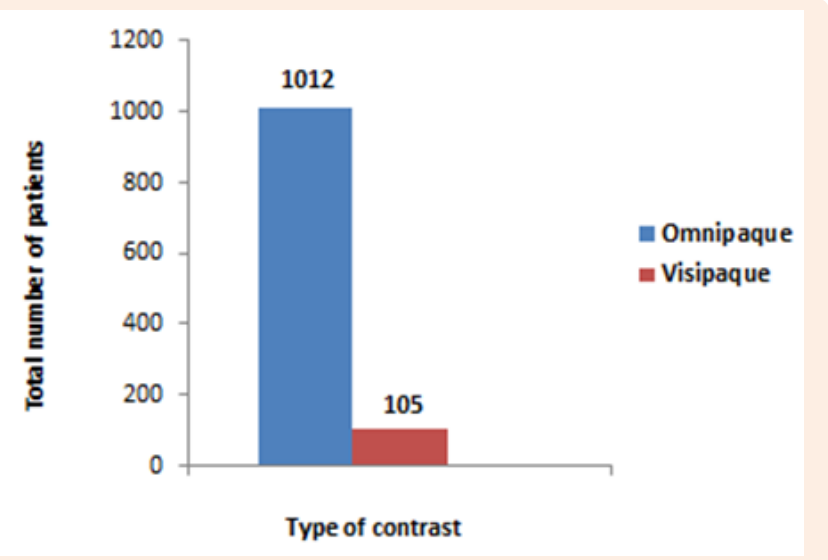

Figure 3: This figure showed that Types of contrast used in these patients, Omnipaque in 1012 and Visipaque in 105 patients.

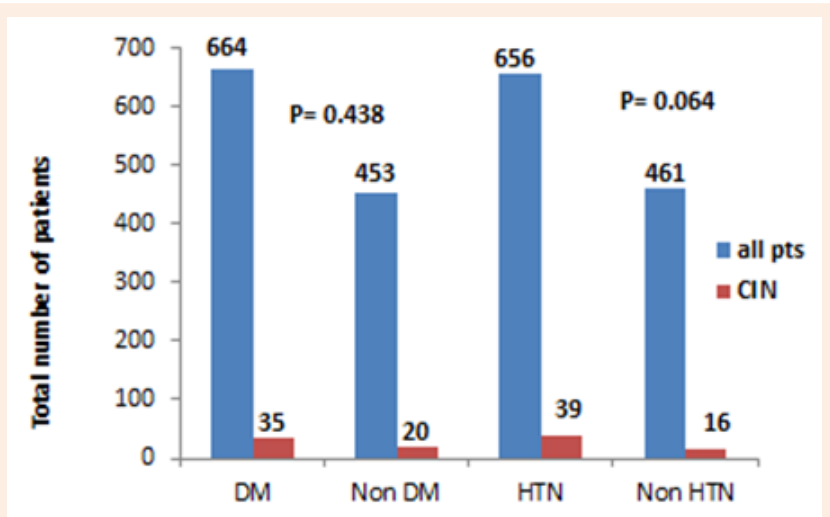

Figure 4: This figure showed the difference in the incidence of CIN between Diabetics, Hypertensive and others.

\section{Discussion}

This study is a single Saudi cardiac center experience, all patients without a contraindication to coronary angiogram 
were included, and the main results showed that the incidence of contrast-induced nephropathy (CIN) was $4.9 \%$, in this small study group. The two major risk factors which precipitate CIN were hypertension and diabetes, with more significance in the hypertensive group. Contrast-induced nephropathy is one of the most common causes of hospital-acquired acute kidney injury. The incidence of CIN varies between 0 and $24 \%$ according to multiple risk factors [5]. It is a transient and reversible form of acute kidney injury. However, the development of CIN is associated with a longer hospital stay, an increased morbidity, and mortality, in addition to a higher financial cost [6].

Contrast-induced nephropathy (CIN) is a known complication of intravenous, iodinated contrast; It is a common cause of renal failure in the inpatient setting [6,7-10]; and is associated with both short- and long-term adverse outcomes [11,12]. Previous reports indicated that CIN occurs in 4 to $20 \%$ of patients after intra-arterial administration after coronary angiography $[13,14]$. Many patients who undergo formal angiography including coronary angiography are admitted in the hospitals, causing a different physiologic status than ambulatory patients. The others, like ED patient, may be more likely to have uncontrolled renal stresses such as undiagnosed or untreated hypertension or chronic hyperglycemia (DM), together with a lower baseline hydration status. Moreover, hospitalized patients in a prolonged supine position may have a lowered rate of renin secretion, resulting in higher renal blood flow [15]. The discussion of the clinical importance of CIN carries out multiple and different opinions. Some of the experts argue that CIN just a laboratorydefined results with a small biologic importance in real practice [16]. Furthermore, the peak sCr level in many patients with CIN is in the normal range or returns to normal within 1 to 3 weeks of exposure [17-20]. Treatment of CIN is mainly supportive, consisting of careful fluid and electrolyte management, although dialysis may be required in some cases [21]. The limitation in the available treatment options makes prevention the cornerstone of management.

Several pharmacological and non-pharmacological approaches have been evaluated for the prevention of CIN. The prevention strategies are most important in patients at high risk for CIN, such as those with AKI or preexisting chronic kidney disease (CKD). It is well established that minimizing the volume of $\mathrm{CM}$ preventing volume depletion and avoiding activation of renal vasoconstriction are the most effective measures to prevent CIN. In addition, the concomitant use of diuretics or nephrotoxins (e.g. nonsteroidal anti-inflammatory drugs (NSAIDs), cytotoxic drugs, and aminoglycosides) should be avoided [5,22].

\section{Conclusion and Recommendation}

The incidence of CIN was $4.9 \%$, and this concludes that hypertension and DM are the major risk factors in Saudi patients, diabetic patients in particular should be closely monitored for CIN and preventive measures should be strictly enforced.

\section{Limitation}

It is a single center study, with a small size sample and no control group for comparison

\section{Acknowledgment}

Special thanks to all catheterization laboratory staff at KACC.

\section{References}

1. Katzberg RW, Lamba R (2009) Contrast-induced nephropathy after intravenous administration: fact or fiction? Radiol Clin North Am 47(5): 789-800.

2. Bartels ED, Baun GC, Gammeltoft A, Gjorup PA (1954) Acute anuria following intravenous pyelography in a patient with myelomatosis. Acta Med Scand 150(4): 297-302.

3. Solomon R (2009) Contrast-induced acute kidney injury (CIAKI). Radiol Clin North Am 47(5): 783-738.

4. Jabara R, Gadesam RR, Pendyala LK, Knopf WD, Chronos N, Chen JP, et al. (2009) Impact of the definition utilized on the rate of contrastinduced nephropathy in percutaneous coronary intervention. Am J Cardiol 103(12): 1657-1662.

5. Perrin T, Descombes E, Cook S (2012) Contrast-induced nephropathy in invasive cardiology. Swiss Med Wkly 142: w13608.

6. McCullough PA, Adam A, Becker CR, Avidson C, Lameire N, et al. (2006) Epidemiology and prognostic implications of contrastinduced nephropathy. Am J Cardiol 98(6A): 5K-13K.

7. Briguori C, Tavano D, Colombo A (2003) Contrast agent-associated nephrotoxicity. Prog Cardiovasc Dis 45(6): 493-503.

8. Schrader R (2005) Contrast material-induced renal failure: An overview. J Interv Cardiol 18(6): 417-423.

9. Hou SH, Bushinsky DA, Wish JB, Cohen JJ, Harrington JT (1983) Hospital-acquired renal insufficiency: A prospective study. Am J Med 74(2): 243-248.

10. Asif A, Preston RA, Roth D (2003) Radiocontrast-induced nephropathy. Am J Ther 10(2): 137-147.

11. Solomon RJ, Mehran R, Natarajan MK, Doucet S, Katholi RE, et al. (2009) Contrast-induced nephropathy and long-term adverse events: Cause and effect? Clin J Am Soc Nephrol 4(7): 1162-1169.

12. Gupta R, Gurm HS, Bhatt DL, Chew DP, Ellis SG (2005) Renal failure after percutaneous coronary intervention is associated with high mortality. Catheter Cardiovasc Interv 64(4): 442-448.

13. Gami AS, Garovic VD (2004) Contrast nephropathy after coronary angiography. Mayo Clin Proc 79(2): 211-219.

14. Rudnick M, Feldman H (2008) Contrast-induced nephropathy: What are the true clinical consequences? Clin J Am Soc Nephrol 3(1): 263272.

15. Raj SR, Biaggioni I, Yamhure PC, Black BK, Paranjape SY, et al. (2005) Renin-aldosterone paradox and perturbed blood volume regulation underlying postural tachycardia syndrome. Circulation 111(13): 1574-1582.

16. Rudnick M, Feldman H (2008) Contrast-induced nephropathy: What are the true clinical consequences? Clin J Am Soc Nephrol 3(1): 263272.

17. Schrader R (2005) Contrast material-induced renal failure: An overview. J Interv Cardiol 18(6): 417-423.

18. Asif A, Preston RA, Roth D (2003) Radiocontrast-induced nephropathy. Am J Ther 10(2): 137-147. 
19. Gami AS, Garovic VD (2004) Contrast nephropathy after coronary angiography. Mayo Clin Proc 79(2): 211-219.

20. Harjai KJ, Raizada A, Shenoy C, Satter S, Orshaw P, et al. (2008) A comparison of contemporary definitions of contrast nephropathy in patients undergoing percutaneous coronary intervention and a proposal for a novel nephropathy grading system. Am J Cardiol 101(6): 812-819.
21. William CM Chui (2010) Contrast-induced Nephropathy. Hong Kong Med Diary 15: 18-19.

22. Solomon,R, Werner C, Mann D (1994) Effects of saline, mannitol, and furosemide to prevent acute decreases in renal function induced by radiocontrast agents. N Engl J Med 331(21): 1416-1420. 